\title{
Contamination control: Removing small particles from increasingly large wafers
}

\author{
A.J. de Jong*a , J.C.J. van der Donck ${ }^{\mathrm{a}}$, T. Huijser ${ }^{\mathrm{a}}$, O. Kievit ${ }^{\mathrm{a}}$, \\ R. Koops ${ }^{\mathrm{a}}$, N.B. Koster ${ }^{\mathrm{a}}$, F.T. Molkenboer, A.M.M.G. Theulings ${ }^{\mathrm{b}}$ \\ ${ }^{a}$ TNO Delft, *ardjan.dejong@tno.nl; \\ ${ }^{\mathrm{b}}$ Delft University of Technology
}

\begin{abstract}
With the introduction of $450 \mathrm{~mm}$ wafers, which are considerably larger than the currently largest wafers of $300 \mathrm{~mm}$, handling with side grippers is no longer possible and backside grippers are required. Backside gripping increases the possible buildup of particles on the backside of the wafers with possible cross-contamination to the front-side. Therefore, regular backside cleaning is required. Three vacuum compatible cleaning methods were selected. Tacky rollers and highvoltage cleaning were selected for particles and plasma cleaning for molecular layers. A test-bench was designed and constructed implementing these three cleaning methods. The first experiments show promising results for the plasma cleaner and the tacky roller.
\end{abstract}

Keywords: Vacuum compatible cleaning, plasma cleaning, tacky rollers, high voltage cleaning, $450 \mathrm{~mm}$ wafers, particle cleaning, cleaning of molecular layers

\section{INTRODUCTION}

\subsection{Overview}

Semiconductor manufacturers continue to push for smaller and cheaper devices, following Moore's Law. In order to maintain this trend, critical dimensions of devices shrink while at the same time wafer size increase. The scale-up of semiconductor wafers is a way to reduce the processing costs during IC production: the same process steps produce roughly twice as many components when wafer size is increased from 300 to $450 \mathrm{~mm}$. European OEMs are busy developing their equipment and processes in line with this trend. Part of the development takes place in the "European Equipment \& Materials Initiative for $450 \mathrm{~mm}$ " (EEMI 450), a European project coordinated by ASMI under the ENIAC research program. Within this European project, TNO is responsible for addressing contamination control issues.

For $450 \mathrm{~mm}$ wafers, side grippers are no longer possible and backside grippers are required. This increases the possibility of particle generation from the backside, which may result in yield losses due to cross-contamination from the backside to the front-side, and carry over to clamps may cause overlay problems [1]. Particles or damage on the back side can cause wafer bending during the lithographic step and in metrology processes.

Within the EEMI 450 project, TNO has built a "test-bench" to study the removal of contamination from the backside of wafers. The aim of this study is to show the feasibility of such a cleaning system in a cluster tool without affecting the throughput of such a tool. Based on the requirements, the three most promising cleaning methods were selected and a "test-bench" was constructed for studying and optimizing the selected cleaning methods.

\subsection{Selection of cleaning methods}

Prior to the design of the test-bench, a selection of cleaning methods was made. The requirements on candidate cleaning methods are:

- $\quad$ Removing organic, oxidic and metallic particles larger than $75 \mathrm{~nm}$ with $90 \%$ efficiency [2]

- Removing molecular layers with a rate of at least $10 \mathrm{~nm} / \mathrm{min}[2]$

- Scalable to High Volume Manufacturing (HVM), i.e. processing in the order of 100 wafers per hour [2]

- Vacuum compatibility

Metrology, Inspection, and Process Control for Microlithography XXVI, edited by Alexander Starikov, Proc. of SPIE Vol. 8324, 832423 - (c) 2012 SPIE · CCC code: 0277-786X/12/\$18 - doi: 10.1117/12.916366 
- No surface damage

The fourth requirement allows the cleaner to be combined with vacuum processing modules. An additional advantage is that organic solvents are then avoided, reducing waste and possible system contamination. This requirement eliminates all cleaning methods requiring liquids or intense gas flows. The fifth requirement eliminates laser ablation and ion sputtering. To satisfy all requirements, three different cleaning methods were selected:

- For molecular layers - Plasma cleaning (cf. [3])

- $\quad$ For particles - Tacky rollers (cf. [4]) and high voltage cleaning (cf. [5])

These methods are implemented in the test-bench.

\subsection{Test-bench design and construction}

Figure 1.1 shows a picture of the test-bench. On top, the wafer holder is visible, carrying a wafer below it. The wafer is transported by a chain, and passes the regions where the three mentioned cleaning systems are positioned. This current test-bench is designed for $100 \mathrm{~mm}$ wafers.

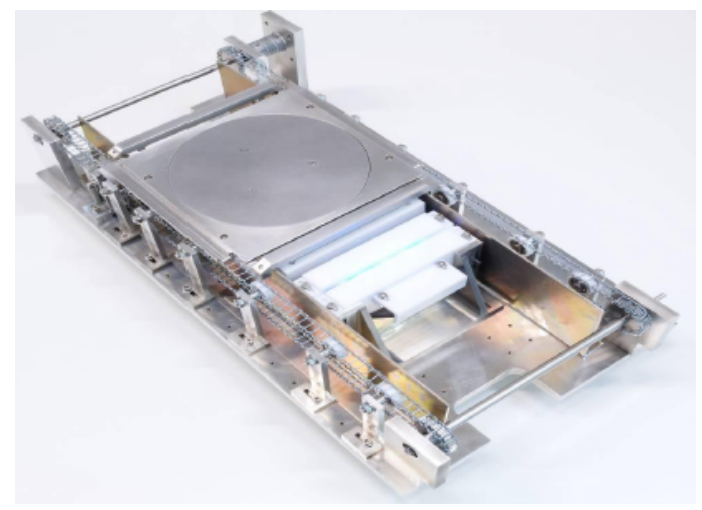

Figure 1.1: TNO test-bench for dry wafer cleaning.

A schematic cross-section of the test-bench is given in Figure 1.2. The wafer (1) is mounted to the holder (2) that is transported by a chain (3). From left to right, the wafer first passes the Tacky Roller (4). The roller is pushed against the wafer by a balancing weight (5). Then, the VUV lamp (6) is passed for photoelectric particle charging for enhanced removal by the high voltage electrode (7). Finally, the wafer passes the plasma chamber (8) where a plasma jet removes molecular layers.

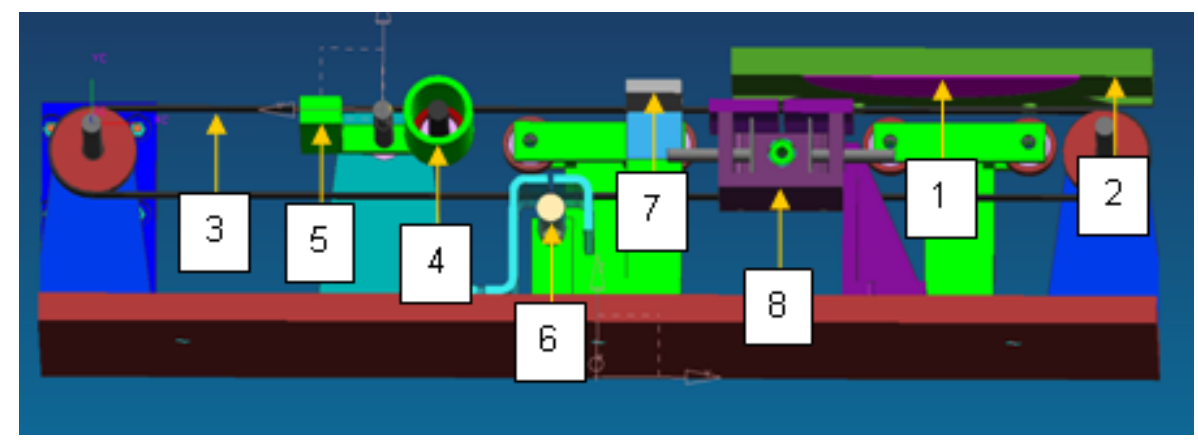

Figure 1.2: Cross-section of the test bench.

An attractive implementation in practice would be to include the cleaner in the load-lock of a vacuum cluster tool, serving various processing modules. Then, every wafer can pass the cleaner when entering and leaving the cluster tool, regularly removing particles and molecular layers. Alternatively, the cleaner could be a separate processing module or could be included in one or more of the other processing modules. 


\section{PLASMA CLEANER}

\subsection{Introduction}

Plasmas are commonly used in industry for etching and deposition. Plasma cleaning is a specific form of plasma etching. Commercial plasma cleaners usually work with $\mathrm{O}_{2}$, Argon or air, but also with $\mathrm{H}_{2}$ for the removal of organic layers from Silicon wafers [3]. The cleaning rate depends on:

- $\quad$ The type of feeding gas

- The ion or plasma density

Particularly efficient plasma cleaners use ICP (Inductively Coupled Plasma) or ECR (Electron Cyclotron Resonance) sources, with ion densities up to about $10^{19} \mathrm{~m}^{3}$. Cleaning rates of up to $0.4 \mathrm{~mm} /$ hour or about $6-7 \mu \mathrm{m} / \mathrm{min}$ are reported for such plasma cleaners [6]. The removal requirement of $10 \mathrm{~nm} / \mathrm{min}$ for molecular is considerably lower, and therefore an RF (13.56MHz) powered CCP (Capacitively Coupled Plasma) source was selected. CCP sources typically have lower plasma densities of $10^{15}-10^{16} \mathrm{~m}^{3}$ [7], but allow the construction of a simple and compact source with homogeneous plasma and surface cleaning.

The procedure of the plasma cleaning experiments was:

- Construct and optimize a stable plasma source that is not or hardly affected by a passing wafer

- Measure the cleaning rate for a well-defined reference sample

- Measure the ion density by a Langmuir probe to characterize cleaning conditions

\subsection{Plasma source optimization}

Figure 2.1a shows a cross-section of the plasma chamber of the CCP source, where the shaded areas are conductors and transparent areas insulators. RF power is applied between the conducting wall and the central electrode. The plasma chamber is fed with a mixture of Argon and air and the gas mixture leaves the top of the chamber through a slit. Thus, sufficient pressure can build up inside the chamber to ignite plasma, while the lower pressure outside leads to a lineshaped plasma jet, cleaning the passing wafer.
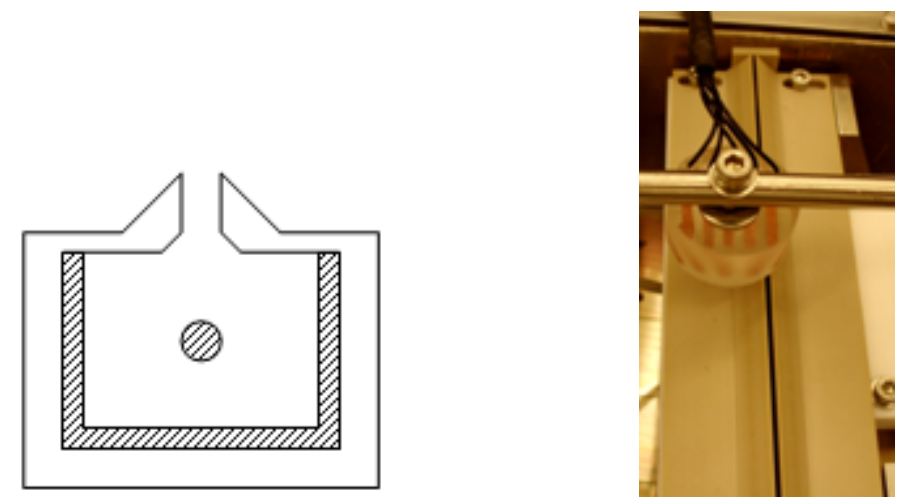

Figure 2.1a-b: Cross-section of the plasma chamber and view from above.

Figure 2.1b shows a picture of the top of the plasma source. Above, the Langmuir probe used for plasma monitoring is visible. The wedges on top of the plasma chamber are added to stabilize plasma flow out of the plasma chamber in the presence of a wafer. The initially designed chamber had a flat top, but in that case, plasma escaped from the edges of the slit when the wafer passed over it. The idea of the wedges is to reduce air flow resistance along the wafer and indeed, with the top of Figures 2.1a-b, a stable plasma jet was obtained over the full width of the slit.

The source is ignited by a $300 \mathrm{sccm}$ Argon flow. Then, an array of purple jets emerges, as shown in Figure 2.2a. The jets move from left to right along the slit with a period of about $1 \mathrm{~s}$. This may be due to local ignition. At the position of a single jet, local viscosity increases, blocking the gas flow towards the jet. Gas flow through to positions with no jet is then enhanced so that the jets continuously displace. Inlet of $30 \mathrm{sccm}$ of air stabilizes the plasma flow along the slit. 
Simultaneously, the color of the jet changes to more orange, as shown in Figure 2.2b. The pressure outside the plasma chamber was about $0.3 \mathrm{mbar}$. The slit dimensions are $3 \mathrm{~mm} \times 10 \mathrm{~cm}$ and the wedges are $6 \mathrm{~mm}$ high at $45^{\circ}$.
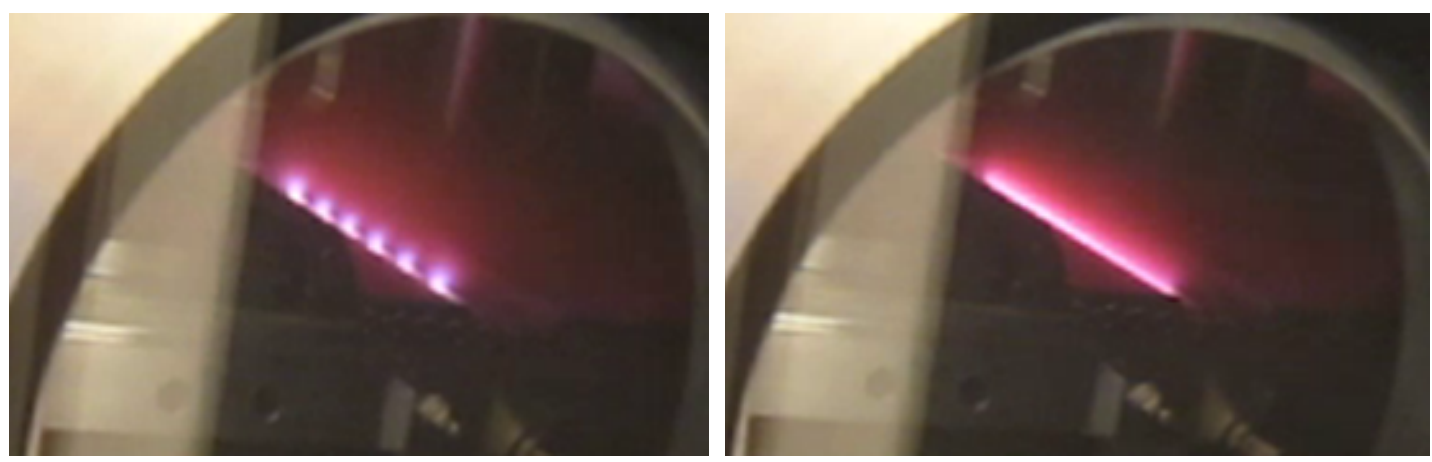

Figure 2.2a-b: Plasma after ignition by Argon and after the inlet of air.

\subsection{Cleaning rate measurements}

In order to quantify cleaning performance, two 3 inch quartz plates with a $20 \mathrm{~nm}$ Carbon coating were cleaned. The plates were exposed to the plasma for 2 and 4 min respectively at a distance of $4.6 \mathrm{~mm}$ above the slit. At this distance, no influence on the plasma jet was noticed. Figure 2.3 shows the sample with 4 min exposure. The Carbon layer is visible as a grey, semi-transparent layer, and the Carbon removal is visible as a vertical bar of a lighter shade of grey. The axial features are part of the sample holder below.

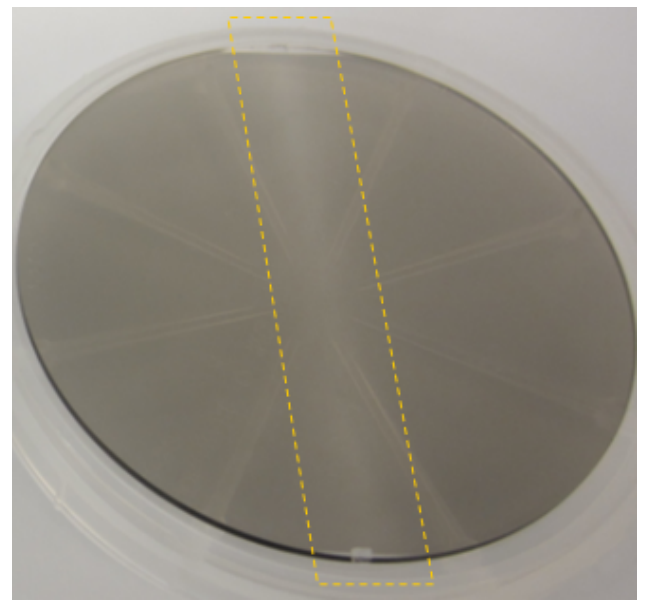

Figure 2.3: Quartz Plates with 20nm Carbon after 4 min of plasma cleaning. The area of maximum Carbon removal is indicated by a dashed parallelepiped.

Carbon removal is measured by a flatbed scanner, providing grayscale maps that can be converted by linear interpolation into Carbon removal maps. Thus, the cleaning rate maps of Figures 2.4a-d were obtained, showing cleaning rates of up to $3-4 \mathrm{~nm} / \mathrm{min}$ above the plasma jet. 

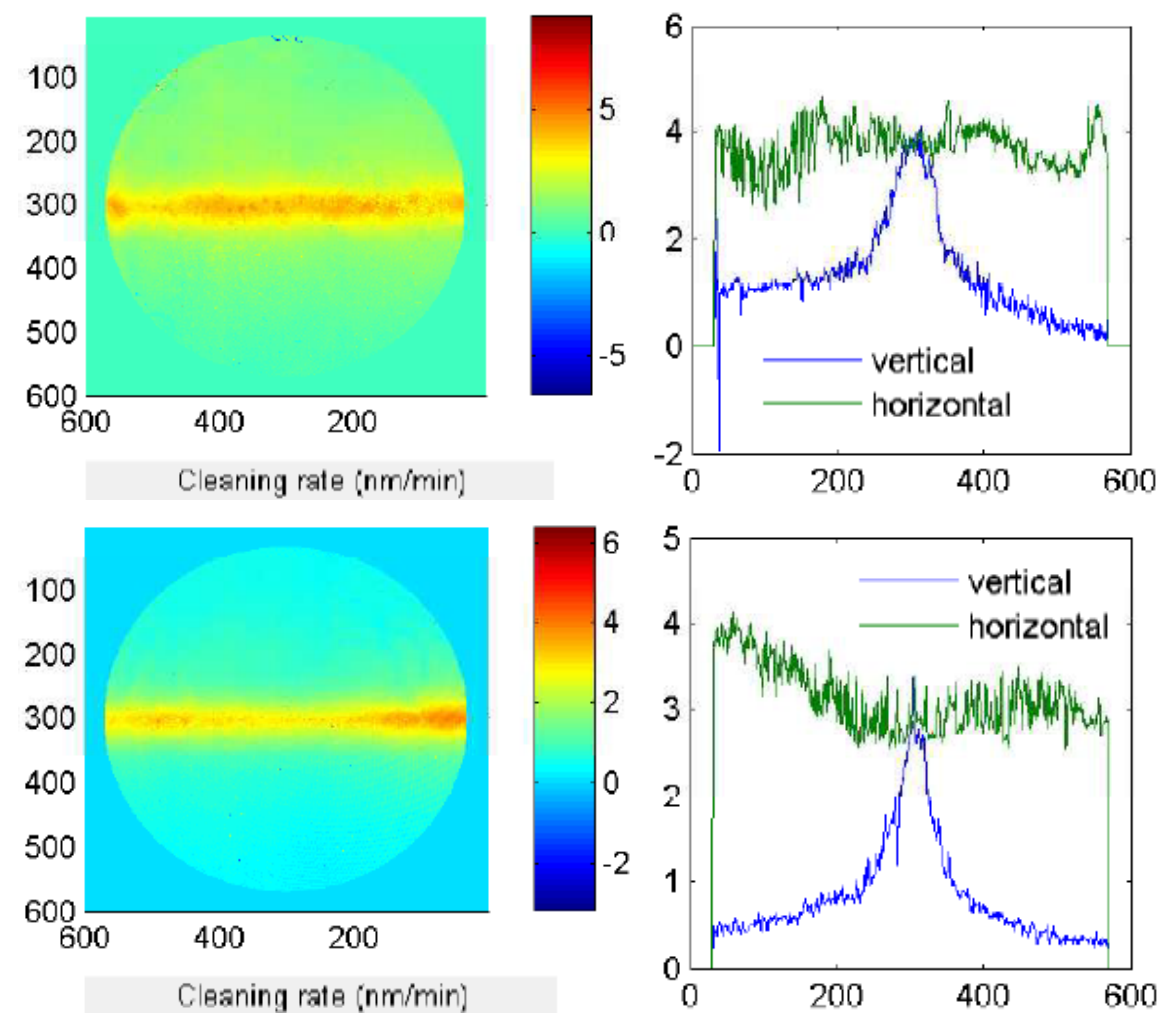

Figure 2.4a-d: Cleaning rate maps in $\mathrm{nm} / \mathrm{min}$ for $2 \mathrm{~min}(\mathrm{a}-\mathrm{b})$ and $4 \mathrm{~min}(\mathrm{c}-\mathrm{d})$ of plasma cleaning. The distance along the 3 inch samples is given in dots with $200 \mathrm{dpi}$ (dots per inch).

\subsection{Plasma diagnostics}

In order to characterize the cleaning conditions of the created plasma, the Langmuir probe of Figure 2.5 was constructed. The probe consists of an array of five Copper strips of $2 \times 14 \mathrm{~mm}^{2}$, casted in epoxy. The probe allows monitoring of plasma density and electron temperature at five positions for single probe measurements, or at four positions for double probe measurements with adjacent pairs of electrodes. The Figure also shows the holder in which the probe is fitted to resemble the geometry of a passing wafer as closely as possible.

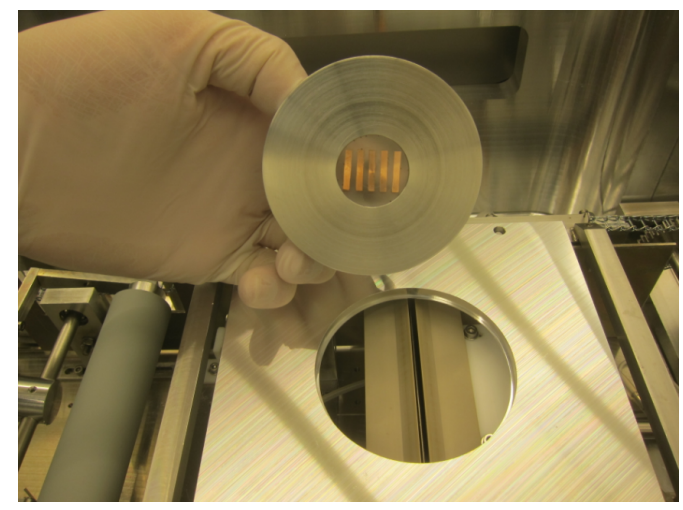

Figure 2.5: Langmuir probe and holder above the plasma source.

With the probe, the bulk plasma density can be estimated from the saturation current for negative voltages (cf. [7]). Electrons are then repelled from the electrode and the current through the probe is a pure ion current:

$$
I_{i}=-q_{e} n_{s} u_{B} A=-q_{e} n_{b} e^{-1 / 2} u_{B} A
$$


Here, $q_{e}=1.6 \times 10^{-19} \mathrm{C}$ is the electron charge, $n_{s}$ is the plasma density at sheath entry, which differs from the bulk plasma density $n_{b}$ by a factor $e^{-1 / 2} \cong 0.61, A$ is the probe area and $u_{B}$ is the Bohm velocity, reading:

$$
u_{B}=\left(\frac{k_{B} T_{e}}{m_{i}}\right)^{1 / 2}
$$

To determine $n_{b}$, the electron temperature $T_{e}$ is needed which is most reliably determined by a double probe. For the symmetric case with identical electrodes, the current-voltage characteristics or $I(V)$ curve reads (cf. [7]):

$$
I=I_{i} \tanh \left(\frac{q_{e} V}{2 k_{B} T_{e}}\right)
$$

At the origin $I=0$ and $V=0$, the slope reads:

$$
\left(\frac{d I}{d V}\right)_{V=0}=\frac{I_{i} q_{e}}{2 k_{B} T_{e}}
$$

The quantities $(d I / d V)_{V=0}$ and $I_{i}$ can be estimated from a measured $I(V)$ curve, so that $T_{e}$ can be obtained from (2.4). With that, $u_{B}$ can be estimated from (2.2) and eventually the plasma density follows from (2.1).

Figure 2.6 shows $I(V)$ curves for different horizontal distances perpendicular to the slit along the $x$-direction, as indicated by the inserted sketch. The blue curve is a double probe measurement with two electrodes symmetrically above the slit of the plasma chamber. From this curve, one can obtain $(d I / d V)=5 \mu \mathrm{A} / \mathrm{V}$, and $I_{i}=36 \mu \mathrm{A} / 2=18 \mu \mathrm{A}$, following the procedure of taking the difference between the asymptotes at high and low voltages [7]. This gives an electron temperature of $k_{B} T_{e}=18 /(2 \times 5)=1.8 \mathrm{eV}$ and a Bohm velocity of $u_{B}=\left(k_{B} T_{e} / m_{i}\right)^{1 / 2}=2060 \mathrm{~m} / \mathrm{s}$. With $A=0.002 \times 0.014=$ $2.8 \times 10^{-5} \mathrm{~m}^{2}$ we get $n_{s}=18 \times 10^{-6} /\left(1.6 \times 10^{-19} \cdot 2060 \cdot 2.8 \times 10^{-5}\right)=2.0 \times 10^{15} \mathrm{~m}^{-3}$ and $n_{b}=n_{s} / 0.61=3.3 \times 10^{15} \mathrm{~m}^{-3}$.

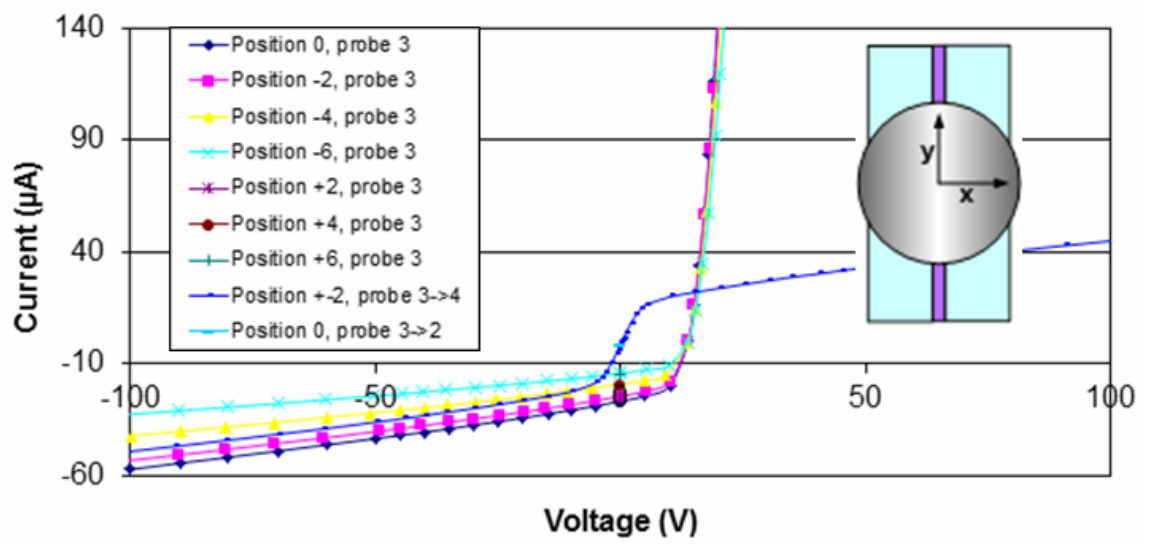

Figure 2.6: Langmuir Probe measurements.

In addition, single probe measurements were done at distances of $x=0,2,4,6 \mathrm{~mm}$ with respect to the slit center. From the asymptotes at negative voltage, ion densities were estimated using the electron temperature of $1.8 \mathrm{eV}$ of the double probe measurement. In Figure 2.7, the estimated ion densities are related to the cleaning rates at corresponding positions. A linear relation would be expected, but the cleaning rate right above the slit, at maximum $n_{b}$, is considerably higher. 


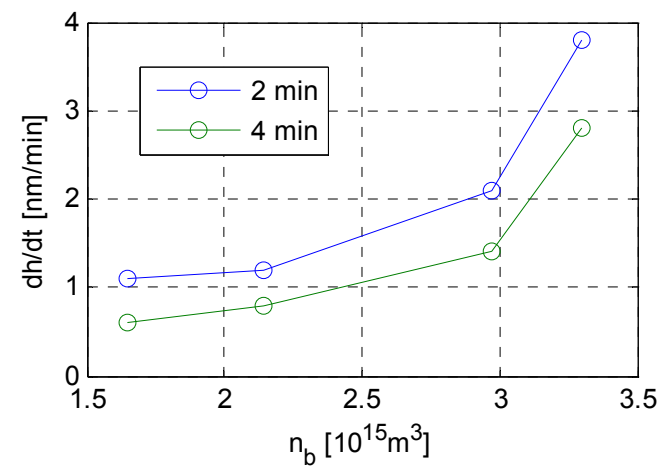

Figure 2.7: Cleaning rate $h$ versus measured plasma density $n_{b}$.

\subsection{Summary and discussion}

After several improvements, the currently operational plasma source provides a stable plasma jet, using an Ar and air mixture as feeding gas. For Carbon coated quartz plates, Carbon removal rates of 3-4 nm/min were obtained right above the slit. This is a factor 2.5-3.0 below the required $10 \mathrm{~nm} / \mathrm{min}$. With the Langmuir probe, the corresponding ion density was estimated as $3.3 \times 10^{15} \mathrm{~m}^{3}$, so that the requirement would be satisfied by increasing the ion density of the plasma jet to about $10^{16} \mathrm{~m}^{3}$. Alternatively, one could:

- Use multiple plasma jets. With the same gas mixture, three line sources would suffice;

- Use a more aggressive gas mixture, such as 50\% Ar with 50\% Oxygen.

This probably leaves enough room for scaling the current plasma cleaner into a cleaner satisfying the removal requirement for molecular layers on $450 \mathrm{~mm}$ wafers.

\section{TACKY ROLLER}

\subsection{Introduction}

Tacky rollers remove particles by a cylinder covered with a sticky polymer, which is rolled over the cleaned surface. In the test-bench, a commercially available tacky roller, the Teknek Nanocleen, DCR hand roller, was included. Figure 3.1a shows a picture of the roller [4]. The chosen roller is vacuum compatible, anti-static and capable of removing $1 \mu \mathrm{m}$ particles. Besides particle size, removal performance may be assumed to depend on particle type, rolling speed and contact pressure. It is also interesting until what extend particle removal is increased by repeated passes.

Besides, there may be an interaction with the plasma cleaner. One may imagine that due to the plasma, remaining solvents may be cracked, reducing the sticking force on the particles.

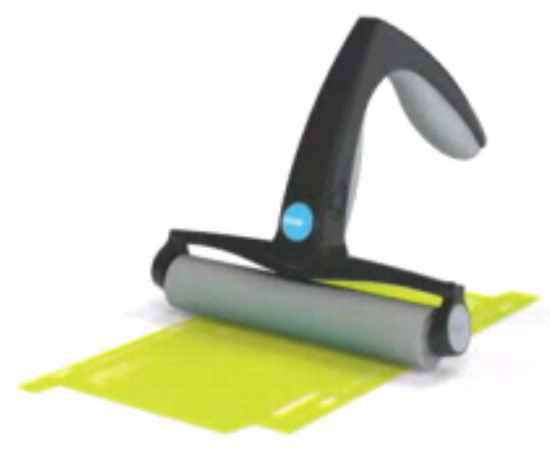

Figure 3.1: Tacky Roller.

The procedure of the tacky roller experiments was: 
- Determine the required particle density for testing the roller in the current system

- Determine the influence of particle size, contact pressure and multiple passes

- Determine the influence of plasma pretreatment

\subsection{Determination of the required particle density}

The minimum particle density is determined by the amount of particles added by the procedure of loading and unloading the wafer into the vacuum chamber, necessary for every particle cleaning experiment. To determine the amount of added particles, a sequence of $10 \mathrm{load} /$ unload cycles was carried out and the numbers of added particles of different sizes is shown in Figure 3.2a-b. The result is obtained by the "RapidNano", an in-house dark field particle scanner [8]. The histogram shows the number of particles of different sizes on an area of $60 \times 60 \mathrm{~mm}^{2}$. In total, 276 particles were added by $10 \mathrm{load} /$ unload cycles, so that each cycle will add about 30 particles of different sizes on the scanned. Although the test-bench is currently not optimized for particles, this is an acceptable result.

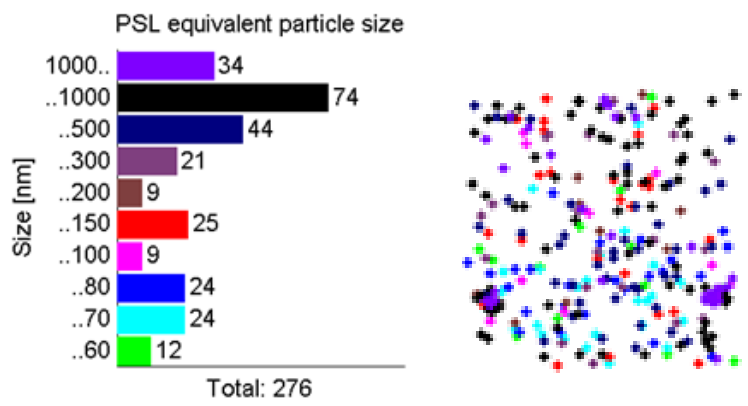

Figure 3.2a-b: Particles added by 10 load/unload cycles.

It follows that with at least 3000 particles per $60 \times 60 \mathrm{~mm}^{2}$, the particle addition of the load/unload procedure is less than under $1 \%$. Therefore, this was the target applied particle density for all particle removal experiments.

\subsection{Particle removal experiments}

Different particle types and sizes were available. PSL (Poly Styrene Latex) particles were selected, as these are expected to be removed more difficultly than oxidic and metallic particles and thus serve as a worst case. PSL particles of $4 \mu \mathrm{m}$ and $900 \mathrm{~nm}$ were used, values around the $1 \mu \mathrm{m}$ specification of the manufacturer. Particles were applied on $10 \mathrm{~cm}$ wafers, polished on both sides, by spinning from an IPA (Iso Propyl Alcohol) solution. All experiments were carried out with a rolling speed of $2.3 \mathrm{~cm} / \mathrm{s}$. This comes down to a cleaning time of about $20 \mathrm{~s}$ for $450 \mathrm{~mm}$ wafers, satisfying the HVM requirement.

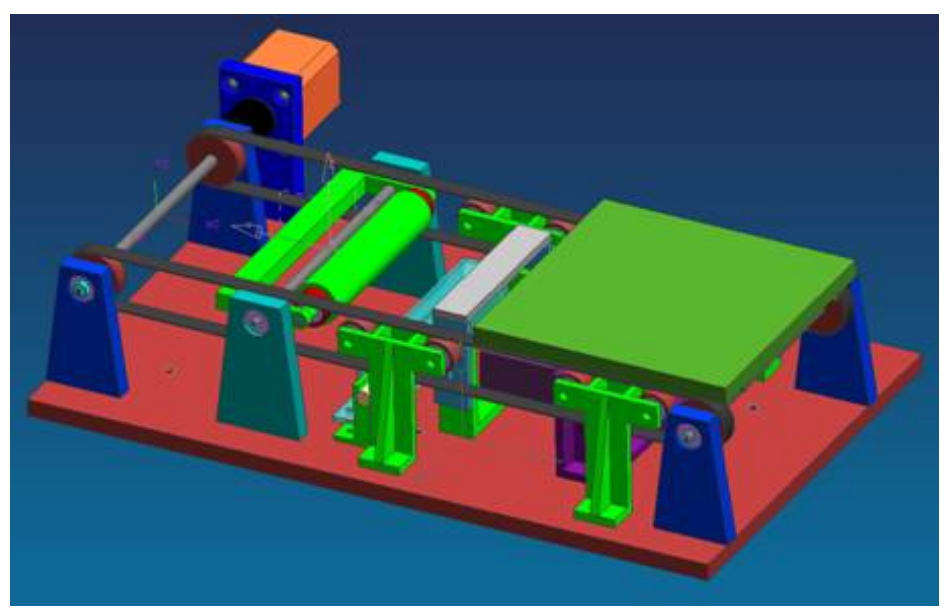

Figure 3.3: Tacky Roller and cantilever system in the test-bench. 
Contact pressure was varied by a cantilever system, visible in the test-bench drawing of Figure 3.3. The tacky roller is the green cylinder on the left and the cantilever is the rectangular bar on the left of it. By adding weight to the cantilever, contact pressure could be increased. Weights of $25 \mathrm{~g}$ and $185 \mathrm{~g}$ were used. The smallest weight is of the same order as the weight of a bare wafer. This situation will probably correspond to the actual case of $450 \mathrm{~mm}$ wafer cleaning.

\subsection{Cleaning results}

Figure 3.4 shows the result for an experiment with $4 \mu \mathrm{m}$ particles and a balancing weight of $25 \mathrm{~g}$. The images were obtained by a dark field particle scanner [9].
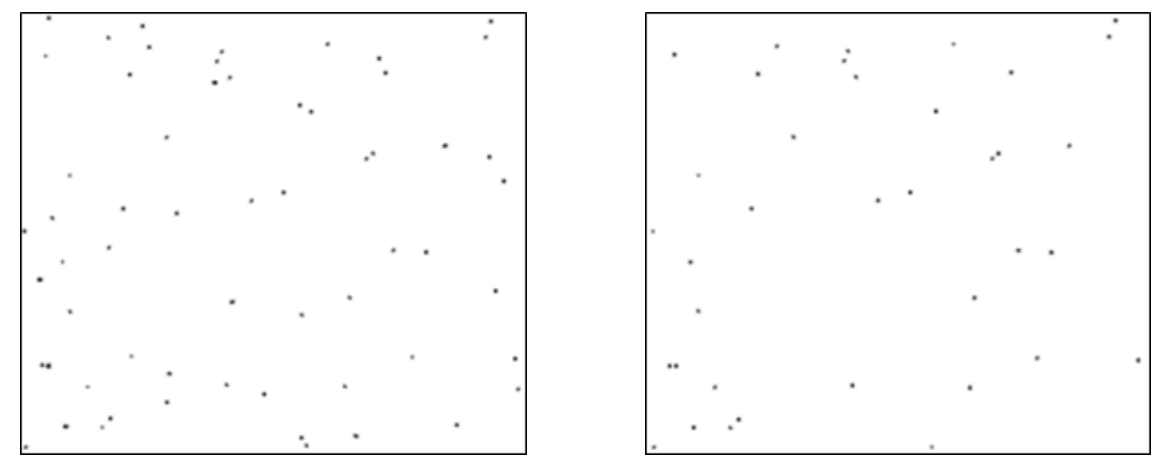

Figure 3.4a-b: PartSens image of $4 \mu \mathrm{m}$ PSL particles before and after a single pass of the Tacky Roller.

Figure 3.5 gives the removed fraction as a function of the number of passes. The blue and green curves are for $4 \mu \mathrm{m}$ PSL particles with $25 \mathrm{~g}$ and $185 \mathrm{~g}$ balancing weights, the red one for and $900 \mathrm{~nm}$ particles. For $4 \mu \mathrm{m}$, particle removal is about $40 \%$ for the first and second passes, with little influence of contact weight. For the smaller weight, the third pass removes about $15 \%$, but the fourth path seems to make no difference. For $900 \mathrm{~nm}$ PSL particles, not more than $5 \%$ was removed for the smaller and larger weights.

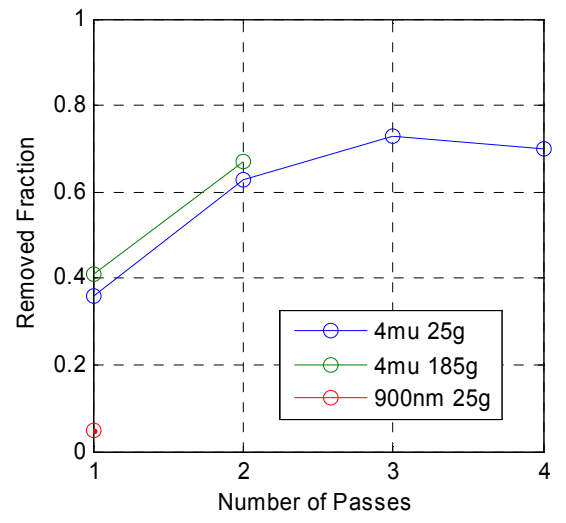

Figure 3.5: Tacky Roller cleaning results.

Finally, an experiment was done to study the influence of plasma exposure. A $10 \mathrm{~cm}$ wafer with $900 \mathrm{~nm}$ particles was passed twice over the plasma source with $2.3 \mathrm{~cm} / \mathrm{s}$, but noticeably different particle removal was not observed. 


\subsection{Summary and discussion}

The current tacky roller removes about $40 \%$ of $4 \mu \mathrm{m}$ PSL particles per pass, which is a good result, preventing particle buildup if the wafers are regularly cleaned. A second pass removes roughly the same percentage. A third pass up to $15 \%$ and a fourth pas seems to make little difference.

Increasing contact pressure from 25 to $185 \mathrm{~g}$ seems to make little difference. For the current experiments with polished wafers, this may not be surprising. For wafers with higher surface roughness, higher contact pressure may help the roller to reach particles in deeper and steeper valleys.

For $900 \mathrm{~nm}$ PSL particles, less than 5\% was removed, more or less in agreement with the manufacturer's specifications.

No significant cleaning improvement was observed by plasma pre-treatment.

\section{HIGH VOLTAGE CLEANER}

\subsection{Introduction}

The idea of particle removal by electric fields is illustrated by Figure 4.1a. Particles on a surface are charged by an electric field between a High Voltage (HV) electrode and the surface. Removal takes place if the electric force dominates the adhesive forces, which are the Van der Waals force, the capillary force and the image charge force.
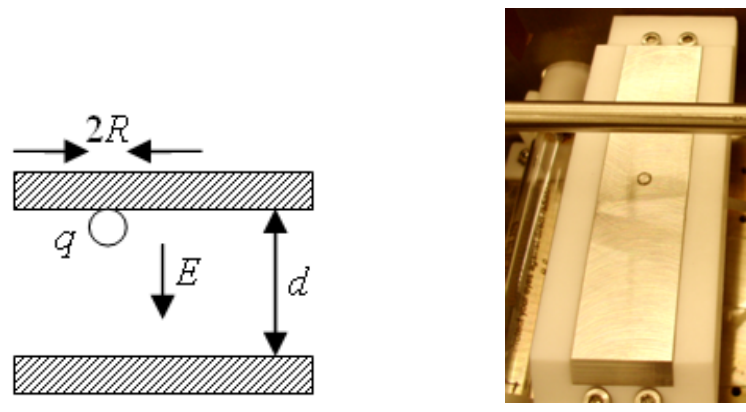

Figure 4.1a-b: Particle removal by high voltage cleaning.

Typical values of electric fields for removing $1 \mu \mathrm{m}$ particles are between $100 \mathrm{kV} / \mathrm{cm}$ and $1 \mathrm{MV} / \mathrm{cm}[5]$. Besides, it may take considerable time to charge particles by the applied electric field. First, the charge must pass the native $\mathrm{SiO}_{2}$ layer of the wafer and secondly, the particle itself must be charged. The time needed to charge a particle made of a material with resistivity $\rho$ and permittivity $\varepsilon$ is the material's relaxation time:

$$
\tau=\rho \varepsilon
$$

For metals, $\rho \cong 10^{-7} \Omega^{-1} \mathrm{~m}^{-1}$, so that with $\varepsilon=8.9 \times 10^{-12} \mathrm{~F} / \mathrm{m}$, the equilibrium charge is rapidly obtained in about $\tau \cong 10^{-18} \mathrm{~s}$. However, for insulators such as $\mathrm{SiO}_{2}$ and PSL, $\rho$ may be as high as $10^{12}-10^{16} \Omega^{-1} \mathrm{~m}^{-1}$, giving $\tau \cong 100-10^{6} \mathrm{~s}$. It then takes an unacceptably long time to charge particles by the electric field from the HVM perspective.

One can think of several ways for charging particles more rapidly than by an electric field. Illumination by VUV was selected, leading to positive particle charging by electron emission via the photoelectric effect. Another option could be to combine the plasma source with the high voltage setup for enhanced particle charging [10].

\subsection{Particle removal experiments}

Similar particle densities were applied as for the Tacky Roller experiments. Figure 4.1b shows the HV electrode, which is a bar of $10 \times 2 \mathrm{~cm}^{2}$, embedded in PTFE to prevent arcing. The HV source can provide $20 \mathrm{kV}$. With the current transport system, the distance between the HV electrode and the wafer could be reduced to $1 \mathrm{~mm}$. In a static situation, the distance could be further decreased to $0.5 \mathrm{~mm}$.

On the left of the electrode in Figure 4.1b, the glass tube of the VUV lamp is visible. This is a commercially available Mercury lamp, the Heraeus GPH150T5VH/4 [11]. This lamp emits $0.4 \mathrm{~W}$ at $185 \mathrm{~nm}$ and $1.5 \mathrm{~W}$ at $254 \mathrm{~nm}$ wavelength. Positive charging during UV illumination continues until the following equilibrium voltage: 


$$
V_{e q}=(h f-W) / q_{e}
$$

Here, $h f$ is the UV photon energy and $W$ is the electron work function of the particle material. For metals, $W=4-5 \mathrm{eV}$, so that they will be charged by the $185 \mathrm{~nm}$ or $6.7 \mathrm{eV}$ radiation up to about $V_{e q}=2 \mathrm{~V}$. For insulators, the work function is half of the band-gap $E_{b}$ plus the electron affinity [12]. For instance, for $\mathrm{SiO}_{2}$ with $E_{g}=9 \mathrm{eV}$ and $E_{a}=1.0 \mathrm{eV}$ [13] one obtains $W=5.5 \mathrm{eV}$, so that $\mathrm{SiO}_{2}$ particles can also be charged.

\subsection{Cleaning results}

Experiments with $4 \mu \mathrm{m}$ PSL and $3 \mu \mathrm{m}$ of Copper particles were carried out. The wafer was passed with $2.3 \mathrm{~cm} / \mathrm{s}$ over the VUV lamp and over the HV electrode at $-2 \mathrm{kV}$ and at a distance of $1 \mathrm{~mm}$. The result for PSL particles is shown in Figures 4.2a-b. No particle removal of both PSL and Copper particles was observed.
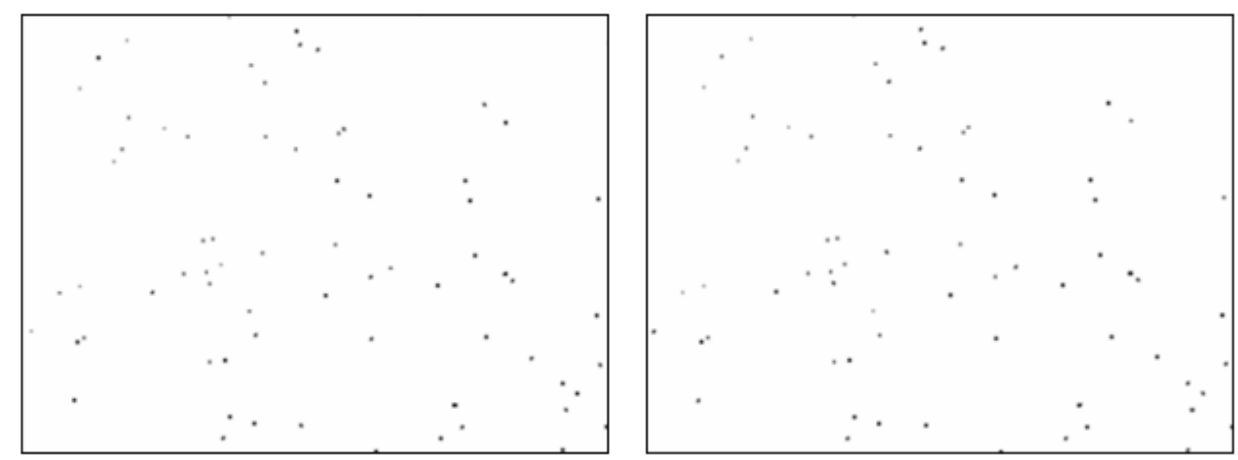

Figure 4.2a-b: $4 \mu \mathrm{m}$ PSL particles before and after HV cleaning.

Two minutes of VUV exposure was still not sufficient to remove particles. Decreasing the distance to $0.5 \mathrm{~mm}$ also did not lead to particle removal.

\subsection{Summary and discussion}

No removal was observed for $4 \mu \mathrm{m}$ PSL and $3 \mu \mathrm{m}$ of Copper particles. For PSL particles, this may be due to the long charging time, but for metallic particles this will not be the reason. The lack of Copper particle removal indicates that the applied electric field was not strong enough.

The electric field could be increased by applying higher voltages or by decreasing the distance between the electrode and the wafer. The first option increases the risk of possible discharges, while the second option is impractical as it would require extraordinary distance control. Therefore, it is not planned to further pursue this cleaning method.

\section{CONCLUSIONS}

A test-bench was designed and constructed to study the feasibility of a vacuum compatible cleaner for the backside of $450 \mathrm{~mm}$ wafers. Three cleaning methods were implemented to fulfill the cleaning requirements: a plasma cleaner for molecular layers, and a tacky roller and a high voltage cleaner for particles.

An RF powered plasma source was constructed and optimized, producing a stable plasma jet with a removal rate of $4 \mathrm{~nm} / \mathrm{min}$ for an Argon and air mixture with a plasma density of $3.3 \times 10^{15} \mathrm{~m}^{3}$. Meeting the $10 \mathrm{~nm} / \mathrm{min}$ requirement could be achieved by increasing plasma density, parallel sources or working with a more aggressive gas mixture.

The tacky roller removes about $40 \%$ of $4 \mu \mathrm{m}$ PSL particles for the first and second passes. The third pass lead to about $15 \%$ of removal and the fourth pass seems to make no difference. Increasing contact pressure did not improve cleaning performance, which may be due to the fact that polished wafers were used. Not more than $5 \%$ for $900 \mathrm{~nm}$ PSL particles were removed, so the minimum particle removal size is between 0.9 and $4 \mu \mathrm{m}$.

The high-voltage cleaner did not remove $4 \mu \mathrm{m}$ PSL or $3 \mu \mathrm{m}$ Copper particles. Not removing metallic particles probably means that the electric fields were too low. 


\section{REFERENCES}

[1] http://www.sematech.org/research/450/docs/ISMI450mmGuidelines.pdf, ISMI 450mm guidelines 2011

[2] http://ismi.sematech.org/docubase/document/450 mm_Equipment Performance Metrics FINAL TT v.2.pdf, ISMI 450mm Equipment Performance Metrics

[3] Mattox, D.M., "Handbook of Physical Vapor Deposition Processing", 2010

[4] http://www.teknek.com/desktop-products.html/der-hand-rollers.html, Teknek, DCR hand roller, 2011

[5] Cooper, D.W., et. al., Aerosol Science and Technology, Vol 13, pp116-123, 1990

[6] Belkind, A., Gershman, S., "Plasma Cleaning of Surfaces", Journal of Vacuum Technology \& Coating, 2008

[7] Lieberman, M.A., Lichtenberg, A.J., "Principles of Plasma Discharges and Materials Processing", 2005

[8] Donck, J.C.J. van der, et. al., "Particle detection on flat surfaces", Proc. Of SPIE Vol. 7969, 79691S, 2011

[9] Advanced Clean Production GmbH, PartSens Operating Instructions, 2007

[10] Lytle, W.M., et. al., "Cleaning of Nanoparticles by Plasma Exposure", in Proceedings of the 2008 International Symposium on Extreme Ultraviolet Lithography, Lake Taho, CA), 2008

[11] http://www.heraeus-noblelight.com/, Heraeus, datasheet GPH150T5VH/4, 2011

[12] http://en.wikipedia.org/wiki/Work function, Wikipedia, work function, 2011

[13] http://inmmc.org/ftp/material/silicon-dioxide.html, Miller, A., Colorado State University, 2011 